\title{
SOME FOOD PLANTS OF HANUMAN LANGUR SEMNOPITHECUS ENTELLUS (DUFRESNE) IN THE WESTERN GHATS OF MAHARASHTRA, INDIA
}

\author{
Sachin A. Punekar \\ Botanical Survey of India, Western Circle, 7, Koregaon Road, Pune, Maharashtra 411001, India.
}

\begin{abstract}
The present study reports 126 flowering plants used as food by Hanuman/Common Langur (Semnopithecus entellus Dufresne) from Western Ghats of Maharashtra. Out of 126 species 94 are reported as food plants for the langurs for the first time.
\end{abstract}

\section{Key words}

Hanuman Langur, Semnopithecus entellus, food plant, plant part

\section{Introduction}

Hanuman Langurs (Semnopithecus entellus) are distributed throughout India, from the Himalaya to the southern tip, except in the deserts, and including Sri Lanka. Within this area about depending on the taxonomist 14 more or less distinguishable races (Prater, 1965), nine species (Groves, 2001) or 11 subspecies (Brandon-Jones et al., in prep.) are recognized. Langurs mainly feed on leaves, flowers and fruits. However, sometimes they also consume insect pupae on leaves and eggs of nesting birds (Rahaman, 1973).

Some details on the observations on the diet of the Hanuman Langurs are provided in this study. Observations made by earlier workers on food plants are no doubt informative but are in fragments, incomplete and mostly restricted to small areas or localities. Present work supplements the data by further field studies in the area under consideration and presents a consolidated botanical checklist of the food plants of Hanuman Langurs from Western Ghats of Maharashtra.

\section{Methodology}

No specific group of Hanuman Langurs was studied for a long period due to academic limitations. As such, visit to any particular area for floristic study on Hanuman Langurs in the Western Ghats of Maharashtra was collateral and thus casual observations only.

The present work is based on visual observations on feeding and scat scanning in different vegetation types between January 1999 to February 2001. Fresh scats were collected and preserved in $10 \%$ formalin for further examination. Each scat mass was immersed in a plastic tray containing water and food materials such as seeds were sorted and identified. Direct observation of langurs feeding on plant parts like leaves, flowers, tender shoots etc. was made in the field.

During the study, attention was given to correct scientific names of food plants (Singh \& Karthikeyan, 2000), family, local name, plant parts consumed, whether native or exotic plants, distribution, habit of food plants and species-specific feeding months of Hanuman Langurs.

\section{Study Area}

The area under study includes different parts of Western Ghats and its adjoining areas of Maharashtra. The study area $\left(14^{\circ} 49^{\prime}\right.$ $-20^{\circ} 25^{\prime} \mathrm{N}$ and $73^{\circ} 01^{\prime}-75^{\circ} 10^{\prime} \mathrm{E}$ ), constitutes about $20,000 \mathrm{~km}^{2}$, which is one-fifth of the gross total area of Maharashtra State.

The climate of western Maharashtra is typically monsoon. The total annual rainfall varies over a wide range with an average of about $200 \mathrm{~cm}$, along the sea face. It may reach to about $600 \mathrm{~cm}$ or even more in the hilly region along the descending western slopes and plains. Rainfall abruptly decreases as one proceeds from the crest to the eastern terrain.

\section{Vegetation}

The vegetation shows a spectrum of variability from west to east. The study area comprises of five broad vegetation types: dry deciduous, moist deciduous, semi-evergreen, evergreen and scrub type of forests (Champion, 1936; Champion \& Seth, 1968).

The dry deciduous type of forest have plant species like Acacia sp., Anogeissus latifolia, Sterculia urens, Terminalia crenulata, Bombax ceiba, Cochlospermum religiosum, Boswellia serrata etc.; the moist deciduous forest contain plant species like Dillenia pentagyna, Careya arborea, Terminalia chebula, Terminalia bellerica, Firmiana colorata, Lagerstroemia

Manuscript received 28 July $2001 \quad$ Revised manuscript received 12 November $2001 \quad$ Accepted for publication 20 April 2002 
Table 1. Enumeration of food plants of Hanuman Langur (Semnopithecus entellus) in the Western Ghats of Maharashtra.

\begin{tabular}{|c|c|c|c|c|c|c|c|}
\hline Botanical name & Local name & Family & $\begin{array}{l}\text { Plant part } \\
\text { consumed }\end{array}$ & $\mathrm{N} / \mathrm{E}$ & $\begin{array}{l}\text { Habit } \\
\text { type }\end{array}$ & Forest & $\begin{array}{l}\text { Feeding } \\
\text { months }\end{array}$ \\
\hline Dillenia pentagyna & Karmal & Dilleniaceae & $\mathrm{TL}, \mathrm{RF}$ & $\mathrm{N}$ & $\mathrm{T}$ & $M D$ & Mar. - Jun. \\
\hline Michelia champaka & Son-chafa & Magnoliaceae & FL, URF & $\mathrm{N}$ & $\mathrm{T}$ & $C$ & Apr. - Sep. \\
\hline Capparis moonii & Waghati & Capparidaceae & RF & $\mathrm{N}$ & $\mathrm{C}$ & MD, SCR & Feb. - May \\
\hline Garcinia indica & Ratamba & Clusiaceae & $\mathrm{LB}, \mathrm{RF}$ & $\mathrm{N}$ & $\mathrm{T}$ & $\mathrm{E}, \mathrm{SE}$ & Feb. - Jun. \\
\hline Garcinia talboti & Phansada, Limboi & Clusiaceae & $\mathrm{RF}$ & $\mathrm{N}$ & $\mathrm{T}$ & $\mathrm{E}$ & Jun. - Aug. \\
\hline Bombax ceiba & Kate Sawar & Bombacaceae & URF & $\mathrm{N}$ & $\mathrm{T}$ & $\mathrm{DD}$ & Apr. - Jun. \\
\hline Ceiba pentandra & Dol Kathi & Bombacaceae & URF & $\mathrm{N}$ & $\mathrm{T}$ & $\mathrm{DD}$ & Feb. - Mar. \\
\hline Sterculia urens & Kandol, Pandruk & Sterculiaceae & S & $\mathrm{N}$ & $\mathrm{T}$ & $\mathrm{DD}$ & Apr. - Jun. \\
\hline Sterculia guttata & Kukar, Golder & Sterculiaceae & $\mathrm{S}$ & $\mathrm{N}$ & $\mathrm{T}$ & MD & Mar. - Jun. \\
\hline Firmiana colorata & Kausi, Supli & Sterculiaceae & $\mathrm{S}$ & $\mathrm{N}$ & $\mathrm{T}$ & MD & Apr. - Aug. \\
\hline Glycosmis pentaphylla & Ambute, Kitmira & Rutaceae & $\mathrm{FL}, \mathrm{RF}$ & $\mathrm{N}$ & ST & SE & Sep. - Jan. \\
\hline Murraya koengii & Karrypatta & Rutaceae & $\mathrm{ML}, \mathrm{RF}$ & $\mathrm{N}$ & ST & $E, S E$ & Mar. - Jun. \\
\hline Murraya paniculata & Kunti & Rutaceae & $\mathrm{ML}$ & $\mathrm{N}$ & ST & $\mathrm{E}$ & All Months \\
\hline Atalantia racemosa & Makad Limbu & Rutaceae & URF, RF & $\mathrm{N}$ & $\mathrm{S} / \mathrm{ST}$ & $\mathrm{E}$ & Dec. - Jun. \\
\hline Garuga pinnata & Kakad & Burseraceae & $\mathrm{RF}, \mathrm{LG}$ & $\mathrm{N}$ & $\mathrm{T}$ & $\mathrm{DD}, \mathrm{MD}$ & Apr. - Jul. \\
\hline Cipadessa baccifera & Gudmei & Meliaceae & $\mathrm{RF}$ & $\mathrm{N}$ & $\mathrm{S}$ & $\mathrm{DD}$ & Oct. - May \\
\hline Aglaia lawii & Burumb, Kat & Meliaceae & $\mathrm{RF}$ & $\mathrm{N}$ & $\mathrm{T}$ & $\mathrm{E}$ & Mar. - Jun. \\
\hline Chloroxylon swietenia & Bhirra, Halda & Meliaceae & $\mathrm{TL}, \mathrm{RF}$ & $\mathrm{N}$ & $\mathrm{T}$ & $\mathrm{DD}, \mathrm{MD}$ & Jun. - Aug. \\
\hline Meytenus rothiana & Henkal & Celastraceae & URF & $\mathrm{N}$ & $\mathrm{S}$ & SE, SCR & Oct. - Jan. \\
\hline Ziziphus mauritiana & Bor & Rhamnaceae & RF & $\mathrm{N}$ & $\mathrm{T}$ & $\mathrm{DD}$ & Aug. - Feb. \\
\hline Ziziphus oenoplia & Burgi & Rhamnaceae & $\mathrm{RF}$ & $\mathrm{N}$ & STS & $\mathrm{DD}$ & Sep. - Mar. \\
\hline Ziziphus rugosa & Toran & Rhamnaceae & RF & $\mathrm{N}$ & SS & SCR & Feb. - Jun. \\
\hline Lannea coromandelica & Shimti & Anacardiaceae & $\mathrm{TL}, \mathrm{RF}$ & $\mathrm{N}$ & $\mathrm{T}$ & $\mathrm{DD}$ & Apr. - Jun. \\
\hline Semecarpus anacardium & Bibba & Anacardiaceae & RF & $\mathrm{N}$ & $\mathrm{T}$ & $\mathrm{DD}$ & Jun. - Sep. \\
\hline Holigarna graphamii & Hulgeri & Anacardiaceae & $\mathrm{RF}$ & $\mathrm{N}$ & $\mathrm{T}$ & $\mathrm{MD}, \mathrm{SE}$ & Apr. - Aug. \\
\hline Spondias pinnata & Ambada & Anacardiaceae & RF & $\mathrm{N}$ & $\mathrm{T}$ & $\mathrm{MD}, \mathrm{SE}$ & Jun. - Oct. \\
\hline Buchanania cochinchinensis & Charoli & Anacardiaceae & $\mathrm{RF}$ & $\mathrm{N}$ & $\mathrm{T}$ & $\mathrm{DD}$ & Apr. - Jul. \\
\hline Mangifera indica & Aamba & Anacardiaceae & URF, RF & $\mathrm{N}$ & $\mathrm{T}$ & $\mathrm{E}$ & Feb. - Sep. \\
\hline Moringa oleifera & Shevga & Moringaceae & S & $\mathrm{N}$ & $\mathrm{T}$ & $\mathrm{DD}$ & Feb. - May \\
\hline Connarus monocarpus & Sundar & Connaraceae & $\mathrm{S}$ & $\mathrm{N}$ & SS & SE, SCR & Jul. - Oct. \\
\hline Mucuna pruriens & Khaj-Kuilee & Fabaceae & S & $\mathrm{N}$ & TW & $\mathrm{DD}$ & Apr. - Jul. \\
\hline Mucunamonosperma & Mothi Khaj-Kuilee & Fabaceae & $\mathrm{FL}$ & $\mathrm{N}$ & TW & MD & Sep. - Jan. \\
\hline Erythrina suberosa & Pangara & Fabaceae & $\mathrm{FL}$ & $\mathrm{N}$ & $\mathrm{T}$ & $\mathrm{DD}$ & Apr. - May \\
\hline Erythina stricta & Kate pangara & Fabaceae & $\mathrm{FL}$ & $\mathrm{N}$ & $\mathrm{T}$ & MD & Feb. - May \\
\hline Butea monosperma & Palas & Fabaceae & $\mathrm{FL}, \mathrm{URF}$ & $\mathrm{N}$ & $\mathrm{T}$ & $\mathrm{DD}$ & Feb. - May \\
\hline Butea superba & Palasvel & Fabaceae & $\mathrm{FL}$ & $\mathrm{N}$ & $\mathrm{L}$ & $\mathrm{DD}, \mathrm{MD}$ & Feb. - Mar. \\
\hline Dalbergia latifolia & Shisham & Fabaceae & TL, URF & $\mathrm{N}$ & $T$ & $\mathrm{DD}$ & Aug. - Sep. \\
\hline Dalbergia horrida var. horrida & Pendkul & Fabaceae & TL, URF & $\mathrm{N}$ & CS & MD, SCR & Jun. - Oct. \\
\hline Pterocarpus marsupium & Bija & Fabaceae & $\mathrm{ML}$ & $\mathrm{N}$ & $T$ & $\mathrm{DD}, \mathrm{MD}$ & Jul. - Feb. \\
\hline Pongamia pinnata & Karanj & Fabaceae & ML, URF & $\mathrm{N}$ & $\mathrm{T}$ & $\mathrm{DD}$ & Sep. - Jan. \\
\hline
\end{tabular}




\begin{tabular}{|c|c|c|c|c|c|c|c|}
\hline Botanical name & Local name & Family & $\begin{array}{l}\text { Plant part } \\
\text { consumed }\end{array}$ & $N / E$ & $\begin{array}{l}\text { Habit } \\
\text { type }\end{array}$ & Forest & $\begin{array}{l}\text { Feeding } \\
\text { months }\end{array}$ \\
\hline Bauhinia vahlii & Chambul & Caesalpiniaceae & URF & $\mathrm{N}$ & $\mathrm{L}$ & $\mathrm{DD}, \mathrm{MD}$ & May - Jun. \\
\hline Cassia fistula & Bahava & Caesalpiniaceae & TL, URF & $\mathrm{N}$ & $\mathrm{T}$ & $\mathrm{DD}$ & Apr. - Jun. \\
\hline Caesalpinia pulcherrima & Shankasur & Caesalpiniaceae & $\mathrm{FL}$ & $E$ & $\mathrm{~S}$ & $\mathrm{C}$ & All Months \\
\hline Leucaena leucocephala & Subhabhul & Mimosaceae & $\mathrm{TL}, \mathrm{S}$ & $E$ & $\mathrm{~T}$ & C & Aug. - Nov. \\
\hline Acacia uriculiformis & Austrelian Babhul & Mimosaceae & $\mathrm{ML}, \mathrm{S}$ & $E$ & $\mathrm{~T}$ & C & All Months \\
\hline Acacia instia & Chilhar & Mimosaceae & $\mathrm{ML}, \mathrm{S}$ & $\mathrm{N}$ & CS & $\mathrm{MD}, \mathrm{SCR}$ & Jun. - Dec. \\
\hline Albizzia lebbeck & Siras & Mimosaceae & $\mathrm{S}$ & $\mathrm{N}$ & $\mathrm{T}$ & $\mathrm{DD}$ & Jun. - Aug. \\
\hline Albizzia odoratissima & Chichva & Mimosaceae & $\mathrm{S}$ & $\mathrm{N}$ & $\mathrm{T}$ & DD & Sep. - Dec. \\
\hline Albizzia procera & Kinhai & Mimosaceae & $\mathrm{S}$ & $\mathrm{N}$ & $\mathrm{T}$ & $\mathrm{DD}$ & Jun. - Sep. \\
\hline Pithecellobium dulce & Vilayatichinch & Mimosaceae & $\mathrm{RF}$ & $E$ & $\mathrm{~T}$ & C & Feb. - May \\
\hline Terminalia bellirica & Behda, Hela & Combretaceae & URF & $\mathrm{N}$ & $\mathrm{T}$ & $\mathrm{DD}, \mathrm{MD}$ & Apr. - Jun. \\
\hline Terminalia chebula & Hirds & Combretaceae & URF & $\mathrm{N}$ & $\mathrm{T}$ & SE & Mar. - Jul. \\
\hline Terminalia crenulata & Ain & Combretaceae & URF & $\mathrm{N}$ & $\mathrm{T}$ & $\mathrm{DD}, \mathrm{SCR}$ & Jul. - Jan. \\
\hline Syzygium cumini & Jambhul & Myrtaceae & $\mathrm{ML}, \mathrm{RF}$ & $\mathrm{N}$ & $\mathrm{T}$ & $\mathrm{E}, \mathrm{SE}$ & Feb. - Jun. \\
\hline Syzygium hemisphgericum & - & Myrtaceae & $\mathrm{ML}, \mathrm{RF}$ & $\mathrm{N}$ & $\mathrm{T}$ & E & Mar. - Jul. \\
\hline Psidium guajava & Peru & Myrtaceae & URF, RF & $E$ & $\mathrm{~T}$ & C & All Months \\
\hline Careya arborea & Kumbha & Lecythidaceae & RF & $\mathrm{N}$ & $\mathrm{T}$ & MD & Apr. - Aug. \\
\hline Memecylon umbellatum & Anjani & Melastomataceae & RFN & & $\mathrm{T}$ & $E, S E$ & Apr. - Jul. \\
\hline Lagerstroemia microcarpa & Nana & Lythraceae & TL, URF & $\mathrm{N}$ & $\mathrm{T}$ & MD & Mar. - Oct. \\
\hline Woodfordia fruticosa & Dhayati & Lythraceae & $\mathrm{FL}$ & & $\mathrm{S}$ & $\mathrm{MD}, \mathrm{SCR}$ & Dec. - May \\
\hline Momordica dioica & Kartul & Cucurbitaceae & URF, RF & $\mathrm{N}$ & $\mathrm{C}$ & $\mathrm{DD}, \mathrm{MD}$ & Aug. - Feb. \\
\hline Solena amplexicaulis & Gometi & Cucurbitaceae & URF,RF & $\mathrm{N}$ & $\mathrm{TR}$ & $\mathrm{DD}, \mathrm{MD}$ & Jul. - Dec. \\
\hline Meyna laxiflora & Alu & Rubiaceae & RF & $\mathrm{N}$ & $\mathrm{S}$ & $\mathrm{DD}, \mathrm{MD}, \mathrm{SCR}$ & Apr. - Jun. \\
\hline Catunaregam spinosa & Gela. Madanphal & Rubiaceae & $\mathrm{RF}$ & $\mathrm{N}$ & $\mathrm{S}$ & $\mathrm{MD}, \mathrm{SCR}$ & May - Jul. \\
\hline Mitragyna parvifolia & Kalamb & Rubiaceae & TL, URF & $\mathrm{N}$ & $\mathrm{T}$ & $\mathrm{DD}, \mathrm{MD}$ & Aug. - Mar \\
\hline Morinda tinctoria & Bartondi & Rubiaceae & $\mathrm{TL}, \mathrm{FL}$ & $\mathrm{N}$ & $\mathrm{TC}$ & $\mathrm{DD}$ & May - Jun. \\
\hline Embelia basaal & Ambat & Myrsinaceae & RFN & CS & $\mathrm{E}, \mathrm{SE}$ & & Feb. - Jul. \\
\hline Embelia tsjeriam-cottam & Ambati & Myrsinaceae & $\mathrm{RF}$ & $\mathrm{N}$ & SS & $\mathrm{MD}, \mathrm{SCR}$ & Jul. - Nov. \\
\hline Embelia ribes & Vavding & Myrsinaceae & $\mathrm{ML}$ & $\mathrm{N}$ & $\mathrm{T}$ & E & All Months \\
\hline Madhuca indica & Moha & Sapotaceae & $\mathrm{FB}, \mathrm{FL}, \mathrm{RF}$ & $\mathrm{N}$ & $\mathrm{T}$ & $\mathrm{DD}$ & Jan. - May \\
\hline Xantolis tomentosa & Katekumbai & Sapotaceae & $\mathrm{RF}$ & $\mathrm{N}$ & $\mathrm{T}$ & MD & Feb. - Jun. \\
\hline Diospyros melanoxylon & Tembhurni, Tendu & Ebenaceae & $\mathrm{RF}$ & $\mathrm{N}$ & $\mathrm{T}$ & $\mathrm{DD}$ & Apr. - Jun. \\
\hline Diospyros montana & Goindu & Ebenacaeae & RF & $\mathrm{N}$ & $\mathrm{T}$ & $\mathrm{MD}, \mathrm{SCR}$ & Mar. - Jul. \\
\hline Jasminum malabaricum & Kusar & Oleaceae & RF & $\mathrm{N}$ & CS & $\mathrm{MD}, \mathrm{SCR}$ & May - Oct. \\
\hline Olea dioica & Parjambhul & Oleaceae & $\mathrm{RF}$ & $\mathrm{N}$ & $\mathrm{T}$ & $\mathrm{E}$ & Jul. - Aug. \\
\hline Carissa congesta & Karvand & Apocynaceae & $\mathrm{RF}$ & $\mathrm{N}$ & $\mathrm{S}$ & $\mathrm{DD}, \mathrm{SCR}$ & Apr. - May \\
\hline Hollarrhena pubescens & Pandhra-kuda & Apocynaceae & ML, TF & $\mathrm{N}$ & $\mathrm{S}$ & $\mathrm{DD}, \mathrm{MD}$ & Jul. - Aug. \\
\hline Cryptolepis buchanani & Kawali & Periplocaceae & ML, TS & $\mathrm{N}$ & CS & $\mathrm{DD}, \mathrm{MD}$ & All Months \\
\hline Cordiadichotoma & Bhokar & Cordiaceae & $\mathrm{RF}$ & $\mathrm{N}$ & $\mathrm{T}$ & $\mathrm{DD}$ & Apr. - Jul. \\
\hline Cordia macleodii & Dahivan & Cordiaceae & $\mathrm{RF}$ & $\mathrm{N}$ & $\mathrm{S}$ & $\mathrm{DD}$ & May - Oct. \\
\hline Ehretia aspera & Datrang & Ehretiaceae & $\mathrm{RF}$ & $\mathrm{N}$ & $\mathrm{S}$ & $\mathrm{DD}$ & Aug. - Feb. \\
\hline Rivea hypocrateriformis & Phanji & Convolvulaceae & $\mathrm{TL}$ & $\mathrm{N}$ & CS & $\mathrm{DD}$ & Jun. - Oct. \\
\hline Dolichandrone falcate & Medshingi & Bignoniaceae & URF, RF & $\mathrm{N}$ & $\mathrm{T}$ & $\mathrm{DD}$ & Jun. - Jan. \\
\hline Heterophragma quadriloculare & Varas & Bignoniaceae & URF, FL & $\mathrm{N}$ & $\mathrm{T}$ & $\mathrm{DD}$ & Feb. - Jul. \\
\hline Stereospermum personatum & Padal & Bignoniaceae & URF & $\mathrm{N}$ & $\mathrm{T}$ & $\mathrm{DD}$ & Aug. - Feb. \\
\hline Gmelina arborea & Shivan, Bharangi & Verbenaceae & $\mathrm{TL}, \mathrm{RF}$ & $\mathrm{N}$ & $\mathrm{T}$ & $\mathrm{DD}$ & Aug. - Feb. \\
\hline Clerodendron serratum & Shivan, Bharangi & Verbenaceae & $M L, F L$ & $\mathrm{~N}$ & $\mathrm{~S}$ & $\mathrm{DD}, \mathrm{MD}$ & Aug. - Sep. \\
\hline Knema attenuata & Rukt-mara & Myristicaceae & $\mathrm{RF}$ & $\mathrm{N}$ & $\mathrm{T}$ & E & Apr. - Oct. \\
\hline Myristica malabarica & Ran-jaiphal & Myristicaceae & RF & $\mathrm{N}$ & $\mathrm{T}$ & $\mathrm{E}$ & Sep. - Dec. \\
\hline Actinodaphae angustifolia & Pisa, Malwa & Lauraceae & $\mathrm{FB}$ & $\mathrm{N}$ & $\mathrm{T}$ & $\mathrm{E}, \mathrm{SE}$ & Nov. \\
\hline Litsea stocksii & - & Lauraceae & $\mathrm{RF}$ & $\mathrm{N}$ & $\mathrm{T}$ & $E, S E$ & Nov. - Jan. \\
\hline Elaeagnus conferta & Ambguli & Eleagnaceae & RF & $\mathrm{N}$ & CS & SCR & Feb. - May \\
\hline Dendropthoe falcate & Bandgul, Betungli & Loranthaceae & RF & $\mathrm{N}$ & $B(P)$ & $\mathrm{DD}, \mathrm{MD}$ & Mar. - Jun. \\
\hline Bridelia squamosa & Aasana, Katak & Euphorbiaceae & $\mathrm{RF}$ & $\mathrm{N}$ & $\mathrm{T}$ & $\mathrm{DD}$ & Oct. - Jan. \\
\hline Phyllanthus emblica & Aawala & Euphorbiaceae & URF, RF & $\mathrm{N}$ & $\mathrm{T}$ & $\mathrm{MD}, \mathrm{SCR}$ & Apr. - Sep. \\
\hline Mallotus phillipinensis & Shendari, Kapila & Euphorbiaceae & URF & $\mathrm{N}$ & $\mathrm{T}$ & $\mathrm{E}, \mathrm{SE}$ & Sep. - Mar. \\
\hline
\end{tabular}




\begin{tabular}{|c|c|c|c|c|c|c|c|}
\hline Botanical name & Local name & Family & $\begin{array}{l}\text { Plant part } \\
\text { consumed }\end{array}$ & $\mathrm{N} / \mathrm{E}$ & $\begin{array}{l}\text { Habit } \\
\text { type }\end{array}$ & Forest & $\begin{array}{l}\text { Feeding } \\
\text { months }\end{array}$ \\
\hline Cleidion spiciflorum & Radkure & Euphorbiaceae & URF, RF & $\mathrm{N}$ & $\mathrm{T}$ & $E$ & Oct. - Jan. \\
\hline Holoptelea integrifolia & Waula, Papadi & Ulmaceae & MF & $\mathrm{N}$ & $\mathrm{T}$ & $\mathrm{DD}$ & Mar. - May \\
\hline Trema orientalis & Ghol & Ulmaceae & $\mathrm{RF}$ & $\mathrm{N}$ & $\mathrm{T}$ & $\mathrm{DD}$ & All Months \\
\hline Morus alba & Tuti & Moraceae & $\mathrm{RF}$ & $E$ & $\mathrm{~T}$ & $\mathrm{C}$ & All Months \\
\hline Ficus bengalensis & Wad & Moraceae & URF, RF & $\mathrm{N}$ & $\mathrm{T}$ & $\mathrm{C}$ & Apr. - Jun. \\
\hline Ficus nervosa & Lothdra & Moraceae & URF, RF & $\mathrm{N}$ & $\mathrm{T}$ & $E, S E$ & Feb. \\
\hline Ficus arnottiana & Pair, Ashta & Moraceae & URF, RF & $\mathrm{N}$ & $\mathrm{T}$ & $\mathrm{DD}, \mathrm{SCR}$ & Feb. - Apr. \\
\hline Ficus hispida & Kala Umbar & Moraceae & URF, RF & $\mathrm{N}$ & $\mathrm{T}$ & MD. SE & Apr. - Jul. \\
\hline Ficus religiosa & Pipal & Moraceae & URF, RF & $\mathrm{N}$ & $\mathrm{T}$ & $\mathrm{DD}, \mathrm{MD}$ & May - Jul. \\
\hline Ficus racemosa & Umbar & Moraceae & URF, RF & $\mathrm{N}$ & $\mathrm{T}$ & $\mathrm{DD}, \mathrm{MD}, \mathrm{SE}$ & All Months \\
\hline Artocarpus heterophyllus & Phanas & Moraceae & URF, RF & $\mathrm{N}$ & $\mathrm{T}$ & E & Feb. - Jul. \\
\hline Gnetum ula & Ombal & Gnetaceae & S & $\mathrm{N}$ & $\mathrm{L}$ & E, SE & Mar. - Nov. \\
\hline Ensete superbum & Rankel, Chowani & Musaceae & URF, RF & $\mathrm{N}$ & $\mathrm{S}$ & $\mathrm{MD}, \mathrm{SE}$ & Jun. - Dec. \\
\hline Dioscrorea pentaphylla & Shendvel & Dioscoreaceae & $\mathrm{FL}$ & $\mathrm{N}$ & TW & $\mathrm{DD}, \mathrm{SCR}$ & Sep. - Oct. \\
\hline Dioscorea oppositifolia & Paspoli & Dioscoreaceae & $\mathrm{FL}$ & $\mathrm{N}$ & TW & $\mathrm{DD}$ & Sep. - Oct. \\
\hline \multicolumn{8}{|l|}{ Asparagus racemosus } \\
\hline var. javanicus & Shatawari, Aaswali & Liliaceae & TS & $\mathrm{N}$ & C & $\mathrm{DD}, \mathrm{MD}$ & Jun. - Sep. \\
\hline
\end{tabular}

TL - tender leaf; ML - mature leaf; LG - leaf gall; LB - leaf bud; FL - flower; FB - flower bud; TS - tender shoot; IF - inflorescence gall; URF - unripe fruit; RF - ripe fruit; S - seed; N - native; E - exotic; T - tree; ST - small tree; S - shrub; B(P) - bushy parasite; C - climber; L - liana; SS - scandent shrub; STS - strangling shrub; CS climbing shrub; TR - trailor; TW - twiner; DD - dry deciduous; MD - moist deciduous; SE - semi evergreen; E - evergreen; SCR - Scrub; C - cultivated

Syzygium cumini, Litsea stocksii, Holigarna grahami etc.; the evergreen type of vegetation is characterized by species like Cleidion spiciflorum, Ficus nervosa, Elaeocarpus glandulosus, Carallia brachiata, Knema attenuata, Myristica malabarica; and the scrub forest vegetation by Capparis moonii, Meytenus rothiana, Connarus monocarpus, Elaeagnus conferta, Terminalia crenulata etc.

\section{Results}

During the present study 126 food plants of Hanuman Langur were reported of which 94 plants are new additions to the existing data of known food plants (Table 1). The diet of the Hanuman Langurs varied from tender leaves to ripe fruits. Hanuman Langurs being an arboreal animal feed mostly on tree species and rarely ground vegetation. It was interesting to note that they also fed on galls of Garuga pinnata, whose leaf galls are epiphyllous, induced by Phacopteron lentiginosum Buckton (Homoptera) (Mani, 1973). Amongst the tree species they mostly fed on the fruits of Ficus, Terminalia, Cordia, Syzygium and Ziziphus species. Amongst plant parts, ripe and unripe fruits were mostly consumed followed by tender leaves, mature leaves, seeds and flowers. Fabaceae, Mimosaceae, Anacardiaceae and Moraceae were the dominant plant families that served as food to the Langurs. Habit-wise food preference are mentioned in Table 2.

\section{Discussion}

The Hanuman Langurs are mainly herbivorous eating plant material such as fruits, seeds, leaves and flowers, with fruits constituting a major part of their diet throughout the year (Prater, 1965).

Among plants, Ficus racemosa, Phyllanthus emblica, Syzygium cumini, Albizia lebbeck, Diospyros melanoxylon, Carissa congesta, Dimocarpus longan, Myristica malabarica, Cleiodion spiciflorum, Eleagnus conferta and Ziziphus species were the major components of their diet.

There is a significant relationship between fruit availability and utilization. Seasonal diet is influenced by fruiting phenology, but it needs further survey in the study area.

Earlier workers like Rahaman and Kankane have enlisted food plants of Hanuman Langur mostly from dry and moist deciduous forests. Rahaman (1973) had listed 41 food plants while Kankane (1978) had listed 35 food plants. In the present study 126 food plants were recognised as food plants of Hanuman Langurs in Maharashtra of which 94 plants are new records (Table 1). They were recorded from different forest types such as dry deciduous, moist deciduous, semi-evergreen, evergreen and scrub forests.

There are direct and indirect benefits of Hanuman Langurs to plants and other animals. They help in seed dispersal of forest trees. The germination percentage of Aglaia lawii seeds present 
Table 2. Habit - wise food preference of Hanuman Langur

\begin{tabular}{ll}
\hline Habit of the plant & No. of species \\
\hline Tree & 79 \\
Small tree & 3 \\
Shrub & 17 \\
Scandent shrub & 3 \\
Climbing shrub & 8 \\
Liana & 3 \\
Climber & 6 \\
Twiner & 4 \\
Trailer & 1 \\
Bushy parasite & 1 \\
Strangling shrub & 1 \\
\hline
\end{tabular}

in the scat of the Hanuman Langur was high compared to the fallen seeds which were collected from the field. The process of digestion and enzymes secreted soften the seeds. Hanuman Langurs consume shoot apices, thus suppressing the apical dominance and facilitating the growth of lateral leaf buds which increase the total biomass of the plant. Deer and wild cattle gather under trees utlized by Hanuman Langurs to eat the fruits and leaves dropped by the langurs.

\section{Acknowledgement}

I am greateful to Dr. R.V. Ranade, Former Head, Department of Zoology, Abasaheb Garware College, Pune for going through the manuscript and suggesting corrections. I am grateful to Research Staff of Botanical Survey of India, Western Circle, Pune; Dr. Subhash Mali, FRLHT, Bangalore; Dr. Tetali, Senior Scientist, NGCPR, Pune; Mr. L.S. Shenitkar, Chief Librarian, Department of Forest, for their kind help. I am thankful to Mrs. Aparna Watve, Mr. Mandar Datar, Mr. Shrinath Kavade and Mr. Mayuresh Paranjape for their timely help.

\section{References}

Brandon-Jones, D., A.A. Eudey, T. Geissmann, D.J. Melnick, J.C. Morales, M. Shekelle and C.-B. Stewart (In prep.). A taxonomy of the Asian primates, a working document from the workshop "Primate taxonomy for the new millennium".

Champion, H.G. (1936). A preliminary survey of the forest types of India and Burma. Indian Forestry Records 1: 286, New Delhi.

Champion, H.G. and S.K. Seth (1968). A Revised Survey of Forest Types of India. Government of India Press, Nasik.

Groves, C. (2001). Primate Taxonomy. Smithsonian Institution Press, Washington and London, 350pp.

Kankane, P.L. (1978). Studies on the Hanuman Langur, Presbytis entellus (Dufresne) at Kanha National Park, pp. 61-71. Proceeding of the workshop on Wildlife Ecology.

Mani, M.S. (1973). Plant Galls of India. The Macmillan Company of India Limited. pp. 61-62.

Prater, S.H. (1965). The Book of Indian Animals. BNHS and PWMWI. Rahaman, H. (1973). The Langurs of Gir Sanctuary (Gujarat). A preliminary survey. Journal of the Bombay Natural History Society, 70(2): 295-314.

Singh, N.P. and S. Karthikeyan (2000). Flora of Maharashtra StateDicotyledons, Vol. I. Botanical Survey of India. 